\title{
New Care Recommendations for Offering Health Care Services to Cancer Patients
}

\author{
Nasim Shams Alizadeh, ${ }^{1}$ Saeid Komasi, ${ }^{2}$ and Ali Soroush, ${ }^{1, *}$ \\ ${ }^{1}$ Lifestyle Modification Research Center, Imam Reza Hospital, Kermanshah University of Medical Sciences, Kermanshah, Iran \\ ${ }^{2}$ Clinical Research Development Center, Imam Reza Hospital, Kermanshah University of Medical Sciences, Kermanshah, Iran \\ "Corresponding author: Ali Soroush, Lifestyle Modification Research Center, Imam Reza Hospital, Kermanshah University of Medical Sciences, Zakarya Razi Boulevard, \\ Kermanshah, Iran. Tel/Fax: +98-8334276299, E-mail: alisoroush.kums@yahoo.com
}

Received 2017 July 22; Revised 2017 September 13; Accepted 2017 October 01.

Keywords: Cancer, Care Service, Psychological Stress, Management

\section{Dear Editor,}

Cancer is one of the most prevalent diseases in developed and under-developed countries (1). It is a major public health problem and is the 3rd leading cause of death in Iran $(2,3)$. The possibility of being life threatening is the main concern of patients in the face of a cancer diagnosis. Most of these patients experience a sense of imminent death and being close to death, therefore, nowadays death anxiety is one of the main psychological factors among the patients with cancer. Even in types of cancers, which have an effective treatment, the patients consider a cancer diagnosis to be equivalent to death due to limited information regarding their disease (4). Despite the medical advances, development of cancer treatments, and also an increasing number of cancer survivors, this disorder causes severe affliction and deep fear in the individual. Death anxiety induces significant emotional and behavioral consequences in individuals, especially those diagnosed with cancer (5, 6 ). This reveals the required attention and designing a comprehensive care plan to lower death anxiety in these patients. Therefore, the purpose of writing this letter is offering new care recommendations for health care services of these patients, especially in order to pay attention to improving their mental health.

The patients may have various defense mechanisms regarding their disease progression; considering the theory by Kubler-Ross, the patients who have terminal diseases experience grief step by step. According to Kubler-Ross, individuals turn to defensive mechanisms in the face of their imminent death. There are 5 defensive mechanisms against death: denial and isolation, anger, bargaining, depression, and eventually acceptance. At the acceptance stage, a patient experiences a mental death and finds that death is inevitable. While a person is recently diagnosed, this disease may be at the stage of denial, anger, or depression. These considerations emphasize the necessity of supplying a suitable and physical environment for hospitalized patients. In other words, cancer patients need a skilled, specialized, and low-cost care at various health centers. The care departments of a cancer hospital include wards, ICU, and motion therapy. There has always been the issue of designing single-bed, double-bed, and multi-bed rooms in hospitals. Certainly, in a cancer treatment center, the best situation is to design single-bed rooms for the patients and their companions. Private treatment rooms were preferred by $50 \%$ of the survey participants, while $28 \%$ always chose a shared space, and $22 \%$ were flexible (7). However, in our country, it seems that there is not a big enough budget, no physical location, and no necessary facilities to provide single-bed rooms for each patient. Therefore, at first, it is better to classify patients according to their disease progression as well as Kubler-Ross grief stages, then assign them to multi-bed rooms.

If this offer is implemented, patients who are in the early stage of their disease are less psychologically affected by end stage patients. In addition to the above suggestions, we recommend the presence of an experienced clinical psychologist in the oncology and radiotherapy departments of health centers. In addition to the psychological support of patients and their relatives, clinical psychologists can play an effective role in choosing the color of rooms, hospital complex, as well as the layout of the rooms. They can also improve the mental health of patients by teaching them to deal with death, anxiety, and death consciousness. Therefore, they can better provide mental health services to terminally ill cancer patients and provide them with the appropriate counseling methods to 
lower death anxiety and achieve a higher quality of life. The above suggestions may be helpful in reducing death anxiety and improving the physical condition of these patients.

\section{Footnotes}

Authors' Contribution: All authors participated in the design of the study, drafted the manuscript, and read as well as approved the final manuscript.

Conflict of Interest: There are no conflicts of interest to report.

Competing Interests: None declared.

Ethical Approval: Not required.

Funding/Support: None.

\section{References}

1. Bektas M, Kudubes AA. Developing scales for the assessment of fatigue in Turkish pediatric oncology patients aged 13-18 and their parents. Asian Pac J Cancer Prev. 2014;15(22):9891-8. [PubMed: 25520124].

2. Farazmandfar T, Janbabaei G, Azadeh H. Common on aspects of cancer. 1st ed. Sari: Mazandaran University of Medical Sciences; 2011.

3. Kolahdoozan S, Sadjadi A, Radmard AR, Khademi H. Five common cancers in Iran. Arch Iran Med. 2010;13(2):143-6. [PubMed: 20187669].

4. Fu MR, Xu B, Liu Y, Haber J. 'Making the best of it': Chinese women's experiences of adjusting to breast cancer diagnosis and treatment. J Adv Nurs. 2008;63(2):155-65. doi:10.1111/j.1365-2648.2008.04647.x. [PubMed: 18537844].

5. Farsi Z, Dehghan Nayeri N, Negarandeh R. Coping strategies of adults with leukemia undergoing hematopoietic stem cell transplantation in Iran: a qualitative study. Nurs Health Sci. 2010;12(4):485-92. doi: 10.1111/j.1442-2018.2010.00563.x. [PubMed: 21210928].

6. Lee A, Wu HY. Diagnosis disclosure in cancer patients-when the family says "no!". Singapore Med J. 2002;43(10):533-8. [PubMed:12587710].

7. Pukszta M. Infusion of evidence: Balancing patient desires with environmental evidence. Oncol Issues. 2017;25(3):24-9. doi: 10.1080/10463356.2010.11883773. 